Loma Linda University

TheScholarsRepository@LLU: Digital Archive of Research, Scholarship \& Creative Works

Loma Linda University Electronic Theses, Dissertations \& Projects

6-2017

\title{
Effect of Image Sharpening on Radiographic Image Quality
}

Jefferson Lee Clark

Follow this and additional works at: http://scholarsrepository.llu.edu/etd

Part of the Prosthodontics and Prosthodontology Commons

\section{Recommended Citation}

Clark, Jefferson Lee, "Effect of Image Sharpening on Radiographic Image Quality" (2017). Loma Linda University Electronic Theses, Dissertations \& Projects. 449.

http://scholarsrepository.1lu.edu/etd/449

This Thesis is brought to you for free and open access by TheScholarsRepository@LLU: Digital Archive of Research, Scholarship \& Creative Works. It has been accepted for inclusion in Loma Linda University Electronic Theses, Dissertations \& Projects by an authorized administrator of TheScholarsRepository@LLU: Digital Archive of Research, Scholarship \& Creative Works. For more information, please contact scholarsrepository@llu.edu. 


\title{
LOMA LINDA UNIVERSITY \\ School of Dentistry \\ in conjunction with the \\ Faculty of Graduate Studies
}

Effect of Image Sharpening on Radiographic Image Quality

by

Jefferson Lee Clark

\begin{abstract}
A Thesis submitted in partial satisfaction of
the requirements for the degree

Master of Science in Prosthodontics
\end{abstract}

June 2017 
(C) 2017

Jefferson Lee Clark All Rights Reserved 
Each person whose signature appears below certifies that this thesis in his/her opinion is adequate, in scope and quality, as a thesis for the degree Master of Science.

Mathew T. Kattadiyil, Professor of Prosthodontics

, Chairperson

Kenneth Abramovitch, Professor of Radiology and Imaging Sciences

Dwight D. Rice, Associate Professor of Radiology and Imaging Sciences

Chandur P. Wadhwani, Assistant Professor of Prosthodontics 


\section{ACKNOWLEDGEMENTS}

I would like to express sincere gratitude to my entire guidance committee for their support, guidance and patience. This is a complex topic requiring dedication, time and understanding for proper navigation of all its facets. I am grateful to Dr. Kattadiyil for introducing me to the topic as well as his indefatigable support. I am also grateful for Dr. Wadhwani whose persisting inquisition into the field of dentistry is directly responsible for the inception of this project and for his continued encouragement. Dr. Rice and Dr. Abramovitch for their voices of reason, moderation and insight into their world of radiology. I am honored to say I was able to work with Dr. Yoon and glean from his experience and thought processes, which made the completion of this project possible. His influence and friendship is valued. I am grateful for Dr. Oyoyo's statistical help as well as his personal sacrifices he gave to make the last minute changes needed.

I would like to thank my wife Christina for showing me understanding and love while raising our five children: Benjamin, Rocco, Zachariah, Emily and Callie. Finally, I would like to thank God whose guidance and blessings allowed me to research a small part of His endless creation. 


\section{CONTENT}

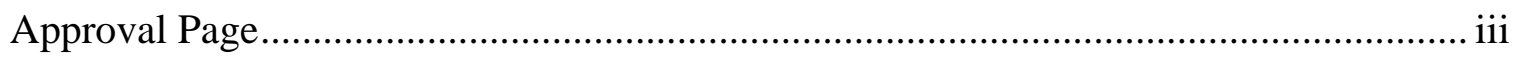

Acknowledgements ........................................................................................................ iv

List of Figures ..................................................................................................... vi

List of Tables ....................................................................................................... vii

List of Abbreviations ............................................................................................. viii

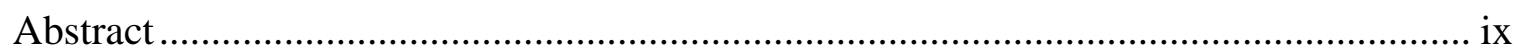

Chapter

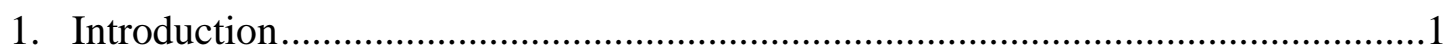

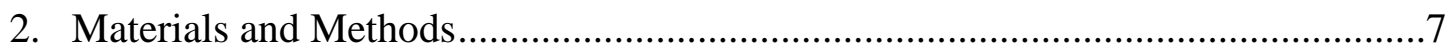

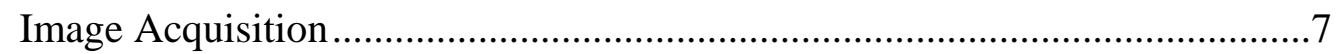

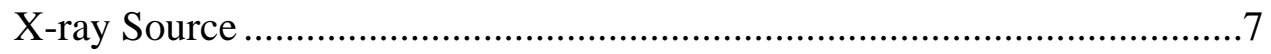

Radiographic Phantom...........................................................................

Imaging Sensors and Software...............................................................

Image Sharpening ....................................................................................

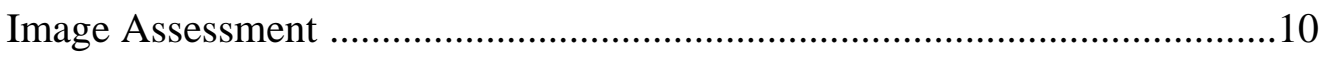

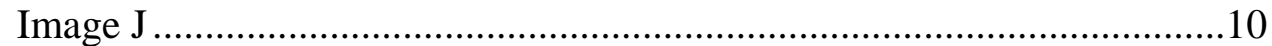

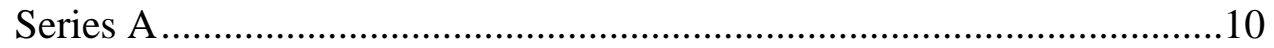

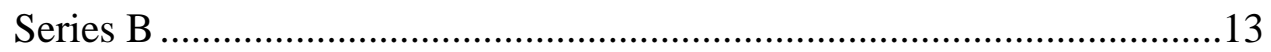

Statistics .....................................................................................15

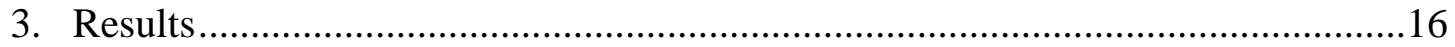

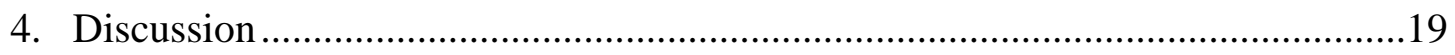

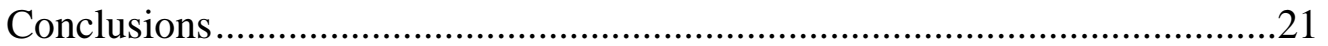

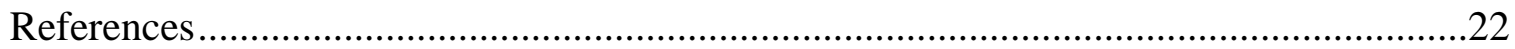




\section{FIGURES}

Figures $\quad$ Page

1. Digital radiograph before and after sharpening .............................................6

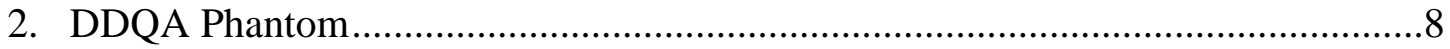

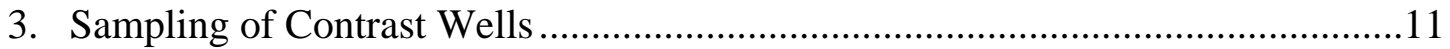

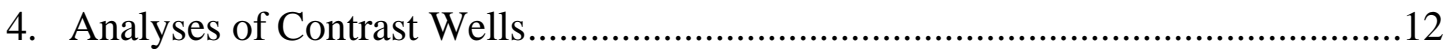

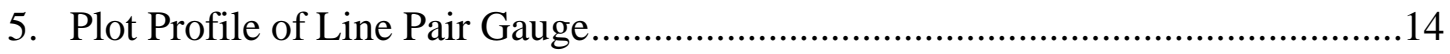

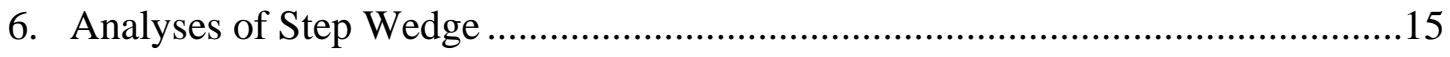




\section{TABLES}

Tables

Page

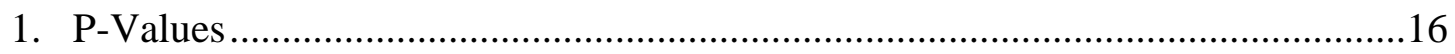

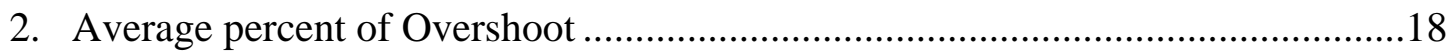




\section{ABBREVIATIONS}

\begin{tabular}{ll} 
ANOVA & $\begin{array}{l}\text { Analysis of Variance } \\
\text { APS }\end{array}$ \\
CCD & Active Pixel Sensor \\
CMOS & Charged-Coupled Devices \\
DDQA & Complementary Metal-Oxide-Semiconductor \\
lp/mm & Digital Dental Quality Assurance \\
PSP & Line Pairs per Millimeter \\
SNR & Photostimulable Phosphor \\
USM & Signal to Noise Ratio \\
\hline
\end{tabular}




\author{
ABSTRACT OF THE THESIS \\ Effect of Image Sharpening on Radiographic Image Quality \\ by \\ Jefferson Lee Clark \\ Master of Science, Graduate Program in Prosthodontics \\ Loma Linda University, June 2017 \\ Dr. Mathew T. Kattadiyil, Chairperson
}

There is evidence that capture and processing of the digital radiographic images may contain artifacts that could reduce the diagnostic value of dental radiographs. This phenomenon has been reported through various case reports. However, its effect on radiographic image quality has not been studied. The purpose of this study is to evaluate the effect of image processing on the quality of dental digital radiographs using the most common type of filter; sharpening. Four imaging modalities (2 CMOS, 1 PSP, 1 conventional film) were used to radiograph a DDQA imaging phantom (Digital Dental Quality Assurance phantom) and to create a flat field radiograph. Contrast resolution, spatial resolution, dynamic range and radiographic noise were assessed for image quality. "Uberschwinger" or overshoot artifact was also assessed. Each image was sharpened and then highly sharpened. Images were compared using Friedman's two-way nonparametric ANOVA. Image quality with regards to noise and resolution were significantly affected by image sharpening. All modalities saw an increase in overshoot artifact when using any level of sharpening. This degree of image sharpening creates artifacts that can be misinterpreted. While image sharpening significantly affects spatial resolution, no significant effect was seen on dynamic range or contrast resolution. 


\section{CHAPTER ONE INTRODUCTION}

Once Wilhelm Conrad Von Röntgen discovered x-rays in 1895, its application to dentistry was recognized almost immediately. (Jacobsohn \& Fedran, 1995) (Raper, 1953) (Sansare, 2014) C. Edmund Kells among others applied this new technology to the field of dentistry. (Jacobsohn \& Fedran, 1995) (Raper, 1953) (Sansare, 2014) Over the years the technology evolved, becoming safer and easier to use. (Sansare, 2014) Today dental radiology is central to oral diagnosis and treatment planning. (Jacobsohn \& Fedran, 1995) (Schweitzer, 2010) (Brettle, 2011) (Affairs, 2006) The introduction of digital radiography has continued this trend, bringing dental imaging into the twenty-first century. Originally, images were captured using analog film and then chemically processed before use. (Affairs, 2006) Today, images are captured electronically using digital sensors and transmitted into viewing software. Two types of digital sensors are used for image acquisition: photostimulable storage phosphor (PSP) sensors, and solid-state electronic sensors. Of the latter, there are two types: charged-coupled devices (CCD) and complementary metal-oxide semiconductor active pixel sensors (CMOS-APS). (Affairs, 2006) (Wenzel, 1998) (Russ, 2016) Digital radiography has proven advantageous over conventional film radiography in various aspects. Digital radiology avoids processing with chemical solutions and reduces exposure to radiation. It also allows faster image display and improves image storage and communication. (Versteeg, 1997) (Schweitzer, 2010) (Brettle, 2011) (Affairs, 2006) (Herrmann, 2012) Another important advantage is the dynamic image it creates with various types of image manipulations such as increasing or decreasing brightness and/or contrast and density inversion. (Versteeg, 
1997) (Affairs, 2006) (Herrmann, 2012) (Wenzel, 1998) Digital intra-oral radiographic images appear to be just as accurate as those of film when diagnosing carious lesions. (Wenzel, 1998)

With digital radiographs, the raw data is usually modified before it can be displayed in a way that is useful to the end user. (Brettle, 2011) (Affairs, 2006) (Russ, 2016) This is because the raw data tends to have sharpness and contrast qualities that are significantly different than film. This modification or processing can include but is not limited to changes in contrast, density, magnification and color. Each sensor manufacturer uses different and proprietary mathematical algorithms for this processing. (Brettle, 2011) Processing occurs at the various stages of image production including the capture, digitization, transmission, calibration and display by the imaging software. (Brettle, 2011) Additional processing considerations occur with the viewing hardware and with image sharing. Each step influences image viewing quality.

There are four primary criteria to physical image quality, namely, contrast resolution, spatial resolution, dynamic range and noise. (Williams, 2007) (Mah, 2011) (Udupa, 2013) (Walker, 2013)

Contrast resolution refers to the ability of the imaging modality to distinguish between differences in radiographic density, specifically the variation in shades of gray between dissimilar objects within the same image. (Lança, 2009) (Williams, 2007) These variations in luminance or gray levels make an object distinct. (Versteeg, 1997) (Russ, 2016) In digital systems, the characteristic response curve of the system is generally linear. In brief, contrast resolution is the smallest difference in brightness that can be detected in the image 
Spatial resolution refers to the ability of the imaging modality (sensor type) to differentiate two objects from one another in an image. (Versteeg, 1997) It is related to the number of pixels per unit length. Thus the size of the pixel plays a role in how clearly proximal objects can be viewed. (Versteeg, 1997) (Versteeg, 1997)

Dynamic range refers to the range of x-ray intensities (radiographic densities) the sensor is able to detect. Dynamic range can be represented by the ratio between the lightest and darkest pixel within the image. (Reinhard, 2010) The bit depth is directly related to this ratio since it limits the number of grays available for each pixel. For example, an 8 bit image is limited to 256 shades of gray. (Versteeg, 1997) As the numbers of bits are increased, the shades of gray increase exponentially.

Radiographic noise refers to random phenomena that obscure the true image. (Russ, 2016) In radiography, there are several random processes like quantum and electrical noise, which is the number of photons that pass unaffected through an object. (a.k.a., shot noise or Poisson noise) (Williams, 2007) These phenomena create a mottled appearance unrelated to the object of interest.

Another parameter for image quality is the distortion of data from digitization and image processing. An example of this is overshoot. (Figure 1.) Commonly referred to by a number of synonymous terms including 'halo', 'Uberschwinger' and 'rebound' artifact. (Brettle, 2011) Unsharp masking (USM) is a standard sharpening tool used in image processing software that creates this artifact. (Brettle, 2011) This is a common algorithm used in many image processing filters. The digitization and image processing that occurs changes the native image data set. This manipulation is often referred to as an 
enhancement but in fact may be degrading the image quality by introducing artifacts such as overshoot that can lead to misdiagnosis. (Schweitzer, 2010) (Brettle, 2011)

A case study by Schweitzer et al. presented a series of radiographs that were processed using the ClearVu ${ }^{\mathrm{TM}}$ feature in Dexis imaging software (Dexis, LLC, Hatfield, PA, USA). ClearVu ${ }^{\mathrm{TM}}$ is an image "enhancement tool, that uses advanced algorithms to further define the image and reveal additional detail and depth.” (Dexis, 2017) This proprietary contrast/sharpening tool is not unlike other such tools provided by other manufacturers. It is designed to improve the diagnoses of dental pathology, including proximal caries, ill-fitting restorations, or fractures. Open margins were perceived when using this feature but not when the feature was turned off leading Sdhweitzer et al. (2010) to conclude the following: (Schweitzer, 2010)

"Contemporary dental digital radiographic software packages often include enhancement features that can alter the visual information presented to a clinician. Careful interpretation of these images is needed as they can potentially result in false-positive diagnoses of dental pathology, prosthesis misfit, and/or structural imperfections within a prosthesis."

Brettle et al. demonstrated the effect this artifact had on clinical diagnosis using a series of 16 radiographs taken from a single panoramic image. These images were scored before and after image processing using the unsharp mask algorithm with the following five point scale of: 1- definitely no pathology, 2- probably no pathology, 3- possibly pathology, 4- probably pathology and 5- definitely pathology. (Brettle, 2011) After USM processing, $89 \%(139 / 157)$ of the diagnoses changed to a diagnosis of greater pathosis. They concluded that there is a potential for image processing artifacts to mimic pathology that cannot be discriminated from genuine pathology. They cautioned that users of digital 
systems should be aware of this artifact and if in doubt, reference should be made to nonrelated high contrast signals and to ultimately refer to the raw data. (Brettle, 2011)

Liedke et al. came to a similar conclusion when studying marginal adaptation of metal restorations with image processing. Liedke et al. stated: (Liedke, 2015)

"Original non-filtered images should be preferred for the assessment of teeth with metal restorations when considering their higher accuracy. High enhancement filters and inversion, particularly, should be avoided in digital images, especially when metal crowns are present."

Although, this issue has been discussed in the dental, medical and veterinarian literature, (Schweitzer, 2010) (Brettle, 2011) (Liedke, 2015) (Tan, 1997) (Oestmann, 1991) (Solomon, 1991) (Jiménez, 2009) (Drost, 2008) little is known about the extent of overshoot artifact created by USM filters on image quality parameters such as contrast resolution, spatial resolution, dynamic range and noise. In addition a series of processing tools are usually made available to the digital dental user. While these filters are options for examining and interpreting radiographic data, they can also unintentionally degrade the image, mimicking genuine pathology. (Brettle, 2011) (Schweitzer, 2010) (Liedke, 2015) It has also been reported how process filters interfere with proper diagnosis of marginal adaptation for teeth restored with metal restorations. (Brettle, 2011) (Schweitzer, 2010) It has not been reported how this phenomenon affects the marginal outlines of dental implants.

In summarizing this review, the capture and processing of radiographic images may contain artifacts that could reduce the diagnostic value of dental radiographs. (Schweitzer, 2010) (Brettle, 2011) (Liedke, 2015) (Tan, 1997) (Oestmann, 1991) (Solomon, 1991) (Jiménez, 2009) (Drost, 2008) However, the effects of image processing on radiographic image quality of various sensors have not been studied. The purpose of 
this study is to evaluate the effect of image sharpening on the 4 quality parameters described above, as well as overshoot.

Null hypothesis: Software image sharpening does not affect image variables such as: contrast resolution, spatial resolution, dynamic range, radiographic noise or overshoot.

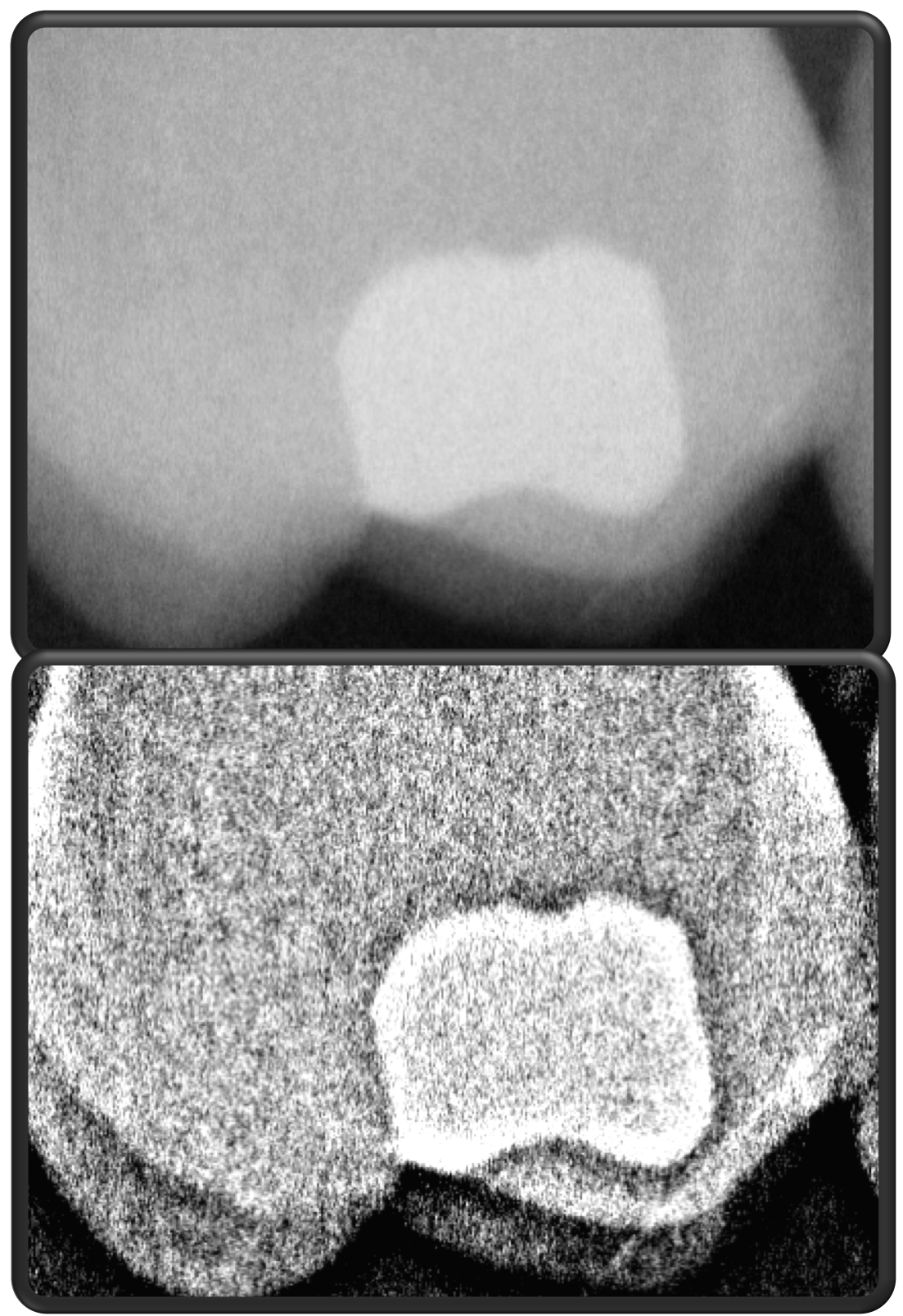

Figure 1. Digital radiograph before and after sharpening 


\section{CHAPTER TWO}

\section{MATERIALS AND METHODS}

\section{Image Acquisition}

\section{X-ray Source}

A Gendex Expert DC (Gendex, Hatfield, PA, USA) x-ray source was used for this study. This direct current unit has a fixed accelerating potential at 65 kilovolts $(\mathrm{kV})$ and a fixed tube current at 7 milliamps $(\mathrm{mA})$. The exposure time has 21 selections ranging from 0.02 to 2.0 seconds. Images were taken and compared between all 21 exposure selections.

\section{Radiographic Phantom}

A Dental Digital Quality Assurance (DDQA) phantom (Dental Imaging Consultants LLC, San Antonio, TX, USA) was used to evaluate three parameters; contrast resolution, spatial resolution and dynamic range. The DDQA consists of two segments: the internal components and a support base. The internal components are comprised of four test gauges; 1 - a series of 6 wells of increasing depth $(0.125-0.75 \mathrm{~mm})$ paralleled by 2 - 6 more wells of diminishing diameters $(0.20-2.5 \mathrm{~mm}), 3$ - a gold foil line pair gauge consisting of 17 line pairs ranging from 5 to 20 line pairs per millimeter $(\mathrm{lp} / \mathrm{mm})$, and 4 - an 1100 grade aluminum alloy step wedge ranging from full attenuation to no attenuation. The support base is comprised of an acrylic stand with two adjustable clamps used to center a variety of imaging sensors beneath the test gauges. Above there are four struts used to support and position the beam indicating device (BID) at a fixed distance. (Figure 2.) 


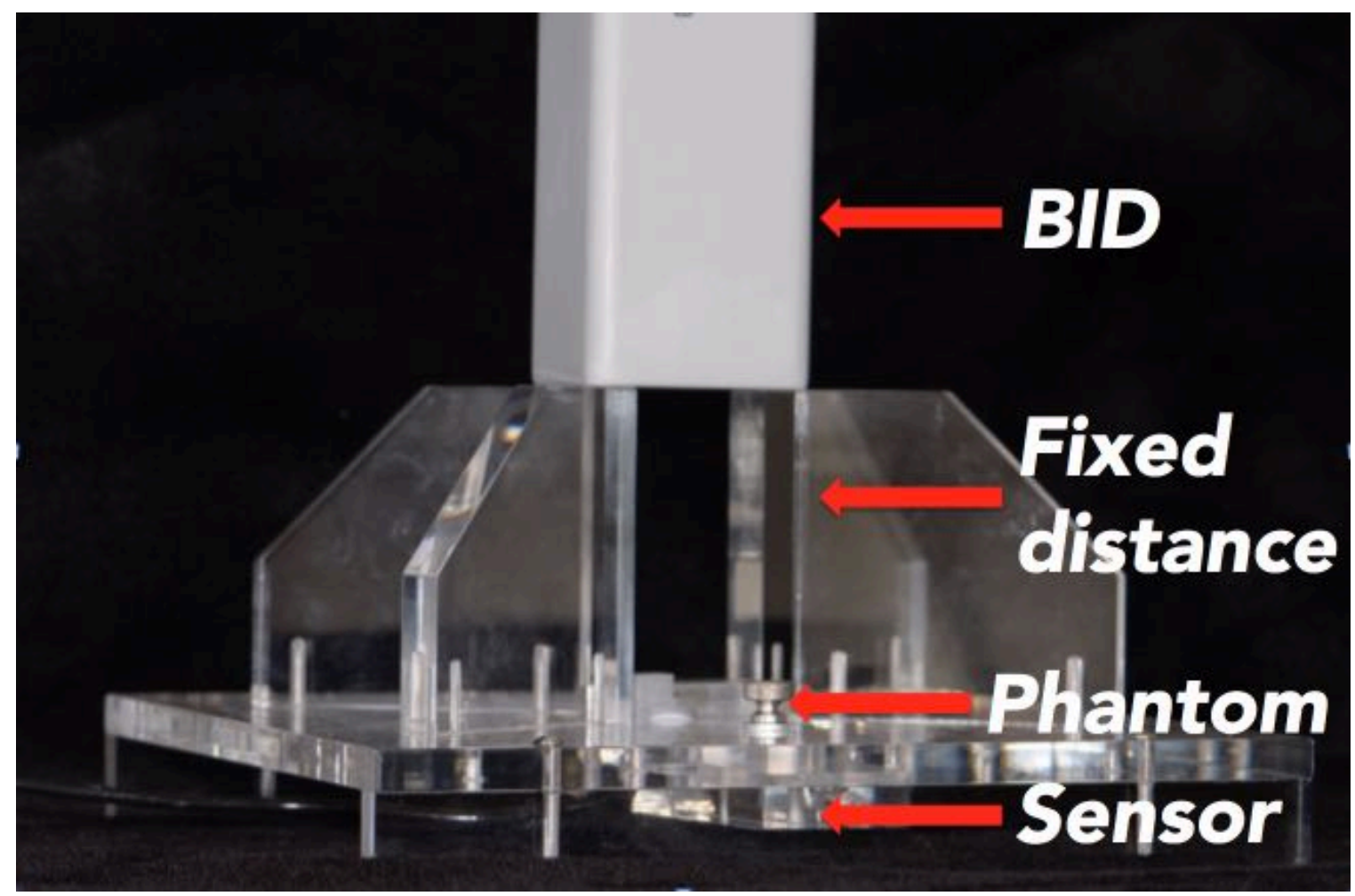

Figure 2. DDQA Phantom

\section{Imaging Sensors and Software}

Four imaging sensors were selected for this study: conventional F speed film size 2 (Insight Dental Film, Carestream Dental LLC, Atlanta, GA, USA), a photostimulable phosphor (PSP) plate system - size 2 (Scan X, Air Techniques Inc., Melville, NY, USA) and two digital size 2 CMOS sensors - (XDR Radiology, Cyber Medical Imagining, Inc., Los Angeles, CA, USA and Schick 33,Dentsply Sirona, Inc., York, PA, USA). The proprietary software was used where available. No sanctioned software was available for film and PSP therefore MiPACS Dental Enterprise Solution software version 3.2.1 (LEAD Technologies Inc., Charlotte, NC, USA) was utilized. XDR Imaging Software 
(Cyber Medical Imagining, Inc., Los Angeles, CA, USA) was used in conjunction with the XDR Anatomic sensor. CDR DICOM 5 (Dentsply Sirona, Inc., York, PA, USA) was utilized with the Schick 33 sensor. A UMAX Powerlook 2100 XL scanner (UMAX Technologies Inc., Dallas, TX, USA) a transmissive photoscanner at 800 dpi, 14-bit gray depth was used to convert the conventional film image to a digital image. A MacBook Pro (Apple Inc., Cupertino, CA, USA) (2.3 GHz Intel Core i7, 8 GB, 1600 MHz, DDR3, HD NVIDIA GeForce GT 650M, 1024 MB, retina screen, resolution 1920 X 1200) running Windows 7 Professional (Microsoft Corporation, Redmond, WA, USA) was used in this study to run the software.

Each imaging sensor was used to create two series (A and B) of 21 images each. Series A was captured using the DDQA phantom and corresponded to the full range of exposure selections from the Gendex Expert DC timer (0.02 to 2.0 seconds). Series B was captured using only the DDQA phantom support base with the internal components removed utilizing the same $\mathrm{x}$-ray source and exposure range. Using the support base without the internal components resulted in a series of flat field images used in noise assessment.

\section{Image Sharpening}

Each image was sharpened then highly sharpened using the sharpening tool in the corresponding software. This consists of sharpening the image to 50\% of the sharpening tool, saving, exporting and then sharpening $100 \%$ of the tool, saving, and then exporting. This resulted in the original image, a moderately sharpened image and a highly sharpened image for a total of 63 images per sensor and 252 total number of images for the study. 


\section{Image Assessment}

\section{Image $J$}

Image $\mathrm{J}$ (National Institutes of Health, Bethesda, MD) is an open source image processing and analysis program used for multidimensional images. Each image was exported from the various software platforms into Image $\mathrm{J}$ software (version $1.50 \mathrm{~b}$ ) using a lossless Tagged Image File Format (.tiff).

\section{Series $A$}

Samples were taken across the six changing depth wells, six changing diameter wells, line pair gauge and step wedge for each image. A modified version of Mah's protocol was used for improved image assessment and standardization. (Mah, 2011) (Udupa, 2013) (Walker, 2013) Settings for sample pixel width and plot range were changed in order to facilitate the analysis. The pixel width for each parameter varied according to the characteristics of each parameter. (Figure 3.) Line width was set at 30 pixels wide for the depth wells, 10 pixels wide for the diameter wells, 200 pixels wide for both resolution and step wedge. A plot profile was created from each sampled data and analyzed. Plot range was changed on the $\mathrm{Y}$ axis from 0 to 200 for contrast wells and 0 to 150 for resolution while the $\mathrm{Y}$ axis for the step wedge remained unchanged. 


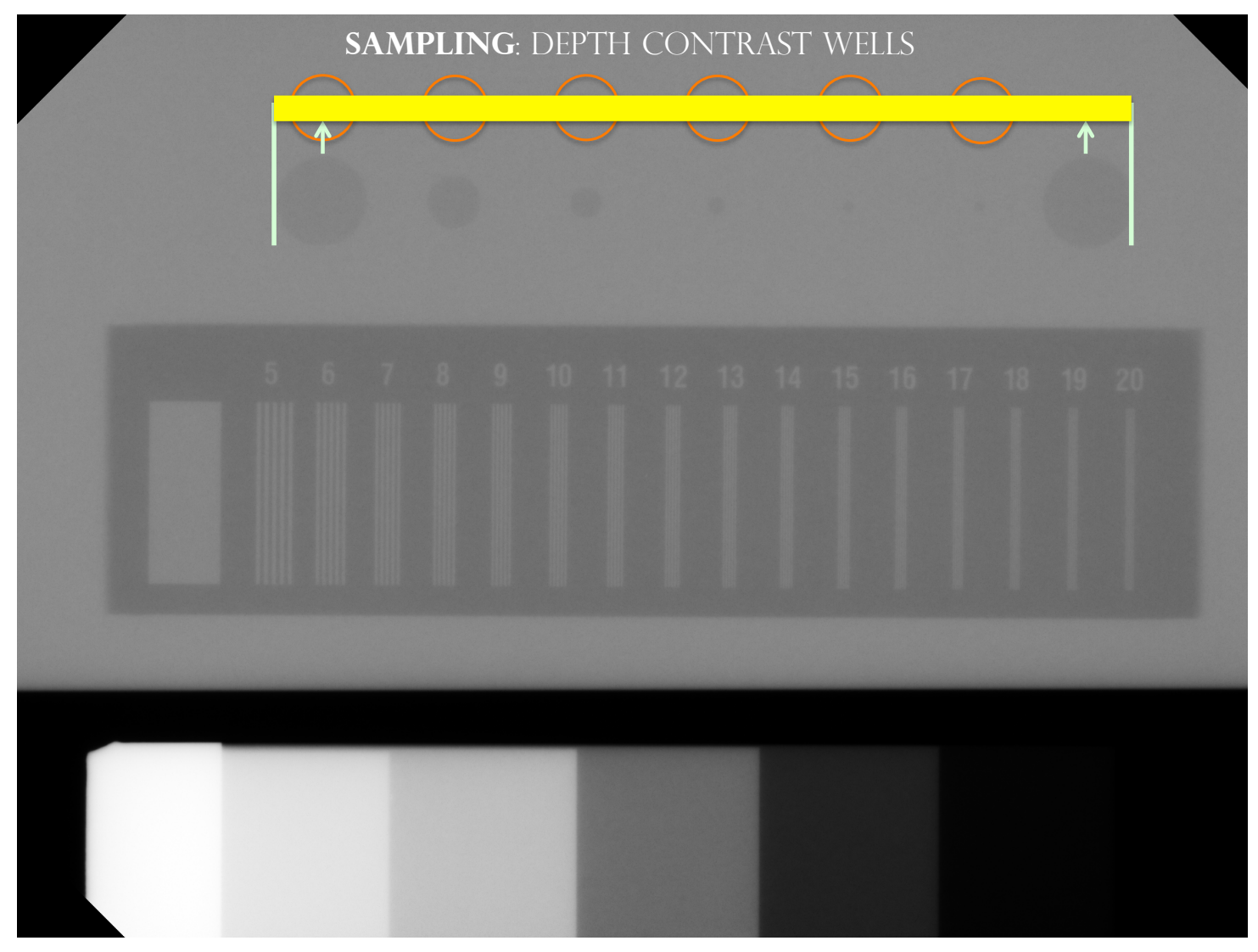

Figure 3. Sampling of Contrast Wells: (depth wells shown increasing depth $(0.125-0.75$ $\mathrm{mm}$ ) Orange highlights wells to be sampled, yellow is the size and shape of the area sampled (30 pixels wide) and green highlights the areas used for orienting the yellow sample bar for consistency.

The sampled data for the depth and diameter contrast wells was obtained as outlined in Figure 3. Standard locations were used within the images to insure consistent comparisons. A plot profile was then created from the sampled data and analyzed.

(Figure 4.) This was done by placing a line across the plot profile, approximating the average inter well (background) gray value. A number was then assigned 0-6 relating to the number of wells that met the following criteria: a) uniform in width and spacing and b) share a uniform visible area beneath line. 


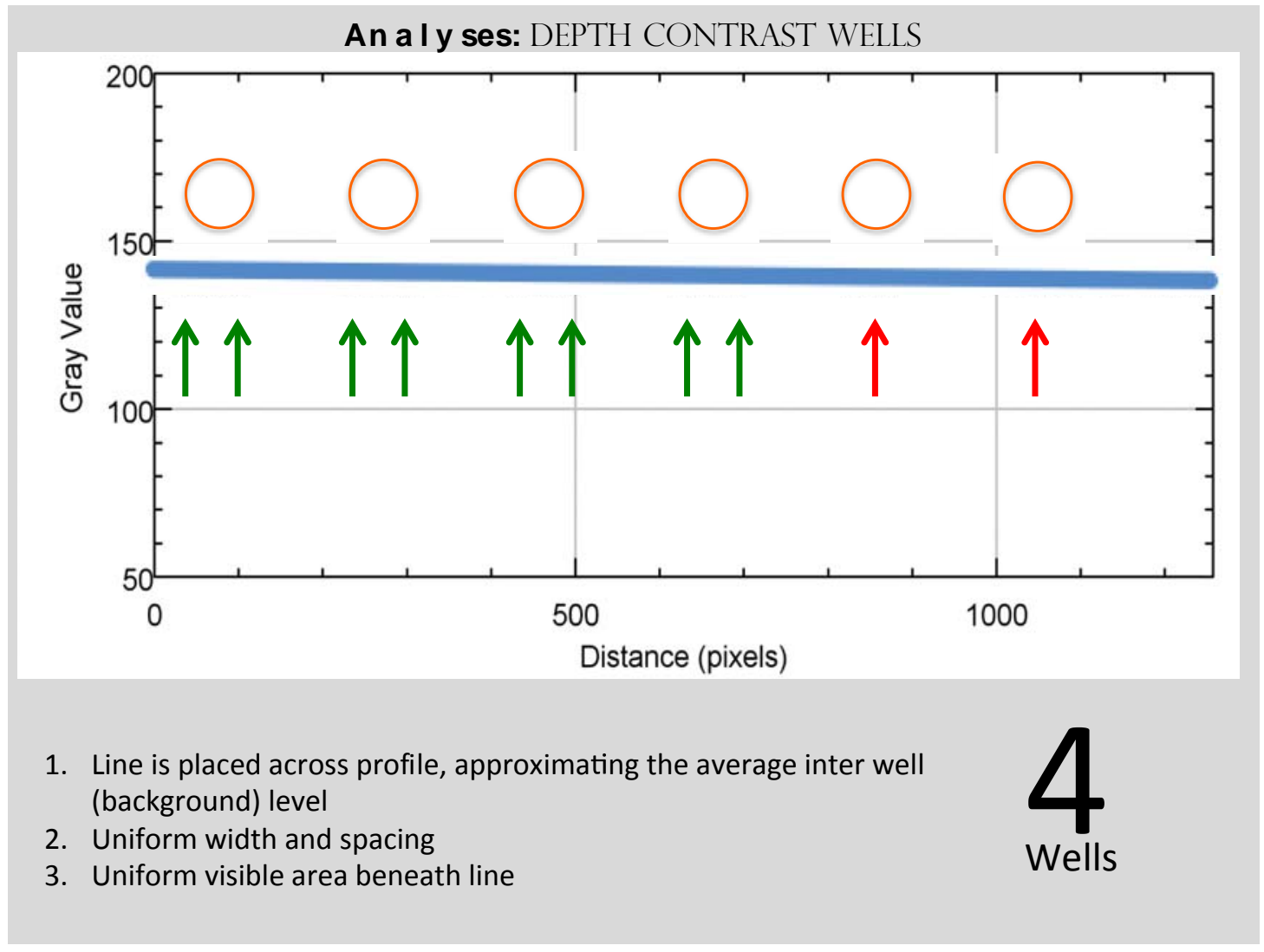

Figure 4. Analyses of Depth Contrast Wells: (depth wells shown increasing depth $(0.125-0.75 \mathrm{~mm})$ ) Orange demonstrates how the wells correlate to the plot, blue is the average inter well line, green met criteria, red failed criteria.

The line pair gauge was split into three sections and sampled individually. Standard locations were used within the images to insure consistent comparisons. Plot profiles were created and analyzed. The highest number of lines pairs/mm were assigned on a scale of 0-20 with the following criteria: a) must see 5 distinguished peaks with 4 valleys and b) A minimum height for each peak was required across the gray scale. The value on the gray scale in measured in increments of 10 . Each peak must cover at least $1 / 2$ of scale, which equals five gray values. (Figure 5.) The image was then assigned the highest number of line pairs that met these criteria. 
Sampling the area across the 7 steps created the data from the step wedge. Standard locations were used within the images to insure consistent comparisons. Plot profiles were created and analyzed. Images were assigned a number between $0-7$ corresponding to the number of visible steps in the plot profile. (Figure 6.)

\section{Series $B$}

This series was taken without the internal components of the DDQA phantom. The purpose of this was to create a flat field image that could be sampled and a signal to noise ratio (SNR) could be calculated and compared. The sample consisted of a $200 \mathrm{x}$ 200 pixel area centered on the image. The data was used to create a histogram and calculate the mean $(\mu)$ and standard deviation. Using the equation $\mathrm{SNR}=\mu / \sigma$ the $\mathrm{SNR}$ was calculated to compare approximated relative noise values. "Mathematically, the SNR is defined as a scalar quantity equal to the ratio of the mean signal to the standard deviation of fluctuations within an image". (Samei, 2003) 

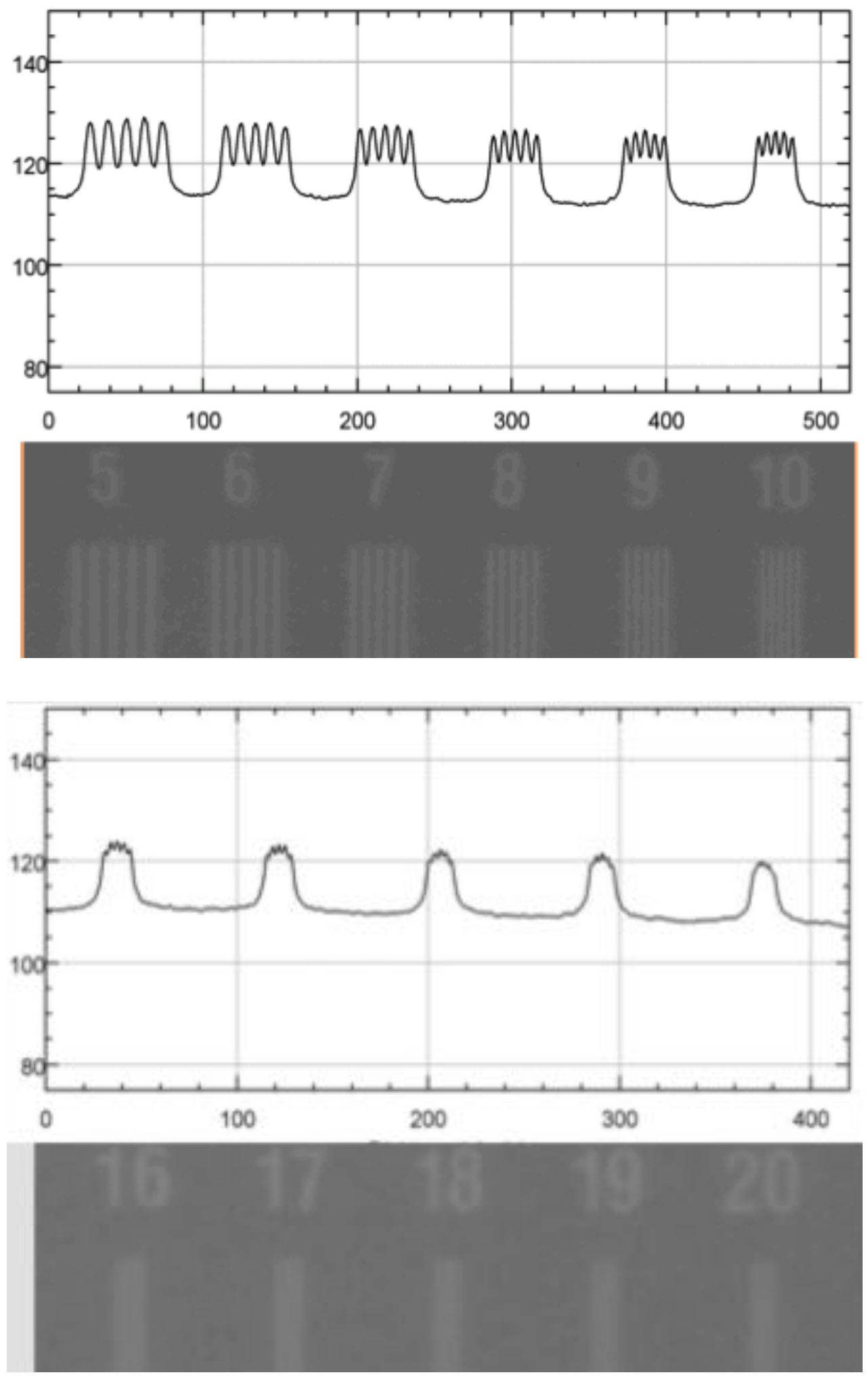

Figure 5. Plot Profiles of Line Pair Gauge: Showing 5-10 lp/mm and 16-20 lp/mm 


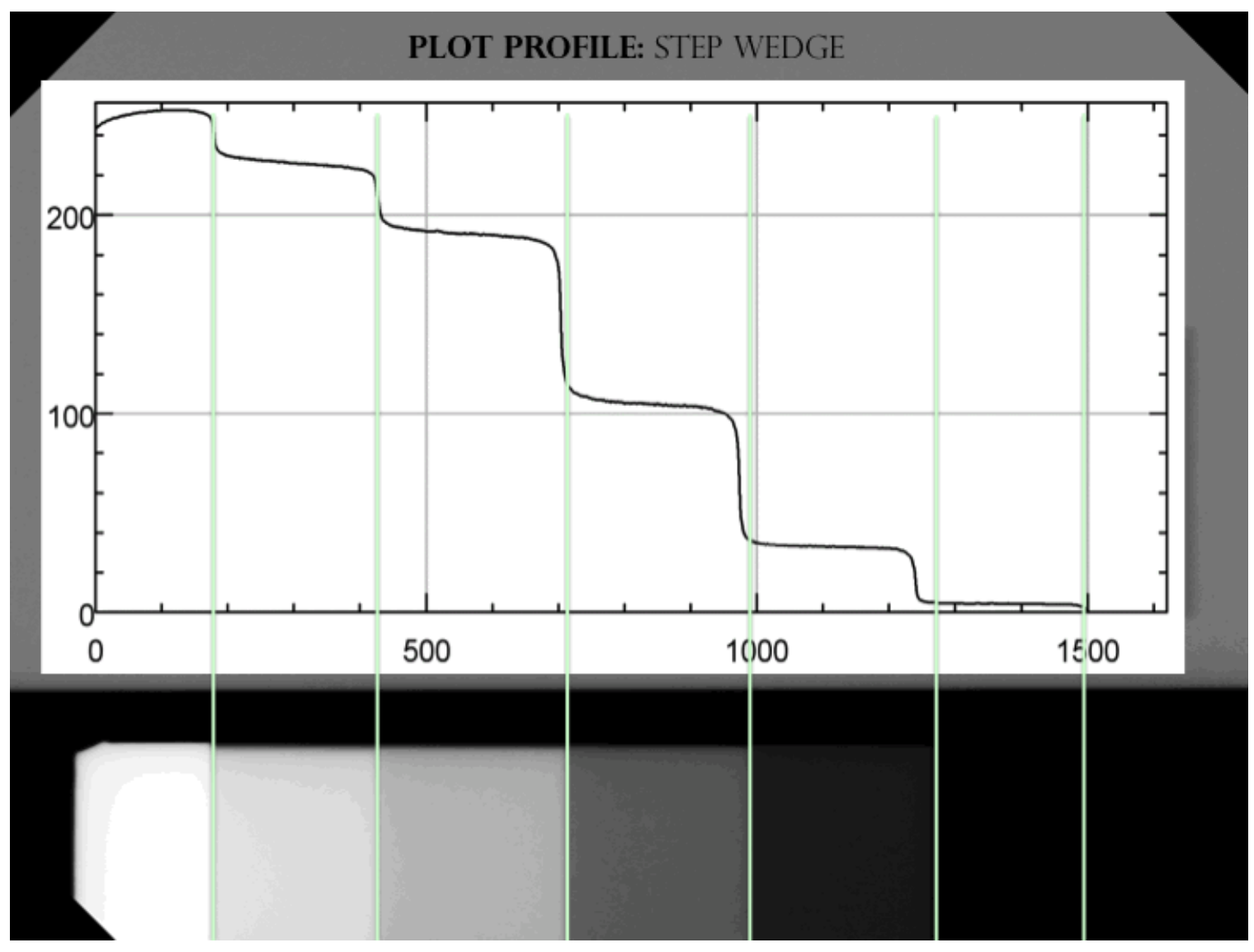

Figure 6. Analyses of Step Wedge

\section{Statistics}

Comparisons were made between the original image, the moderately sharpened image and the highly sharpened image for each group in both series using a Friedman's two-way nonparametric ANOVA analysis. An $\alpha$ level of 0.05 was selected. 


\section{CHAPTER THREE}

\section{RESULTS}

The dynamic range was the most consistent testing variable across all sensors (average $p$ value .578). It was the least affected by image sharpening. This was followed by both series of contrast depth and diameter wells (average p value of .149 and .258 respectively). Both sets of contrast wells demonstrated some degradation at lower exposure levels. Image quality with regards to noise $(\alpha$ level $=0.000)$ and Resolution $(\alpha$ level $=0.000)$ were significantly affected by image sharpening. (Table 1. )

Table 1. p-Values

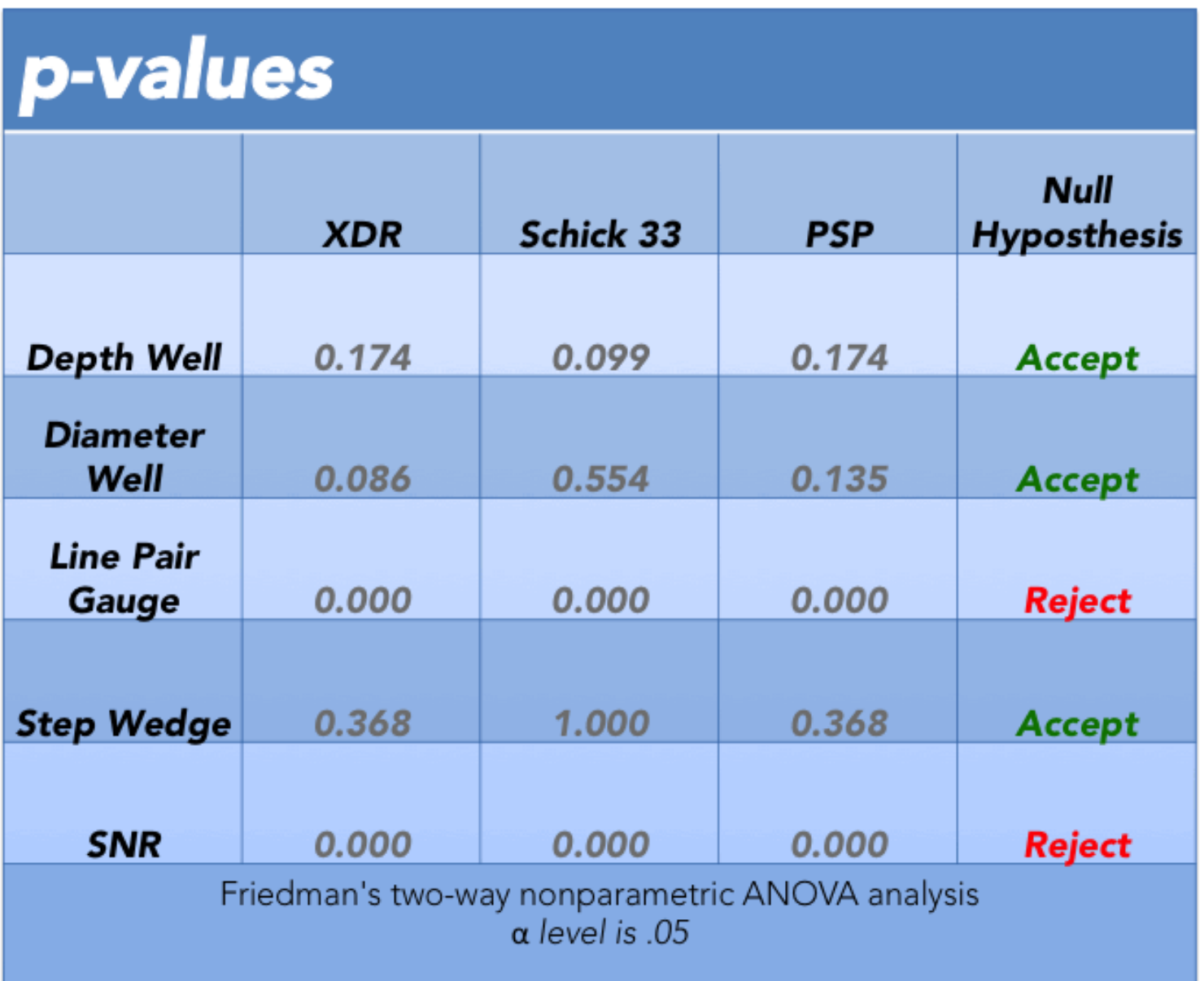


Moderate sharpening improved the resolution on average of $1.287 \mathrm{lp} / \mathrm{mm}$ for XDR, .333 $\mathrm{lp} / \mathrm{mm}$ for PSP and $3.476 \mathrm{lp} / \mathrm{mm}$ for Schick 33 . When sampling from the middle of the range XDR and PSP averaged $.909 \mathrm{lp} / \mathrm{mm}$ while Schick 33 improved on average 3.818 1p/mm. XDR demonstrated the best overall resolution, however the image was significantly degraded when highly sharpened by an average of $2.285 \mathrm{lp} / \mathrm{mm}$ or 3.182 $1 \mathrm{p} / \mathrm{mm}$ when sampling from the middle of the exposure range. Both PSP by $(.667 \mathrm{lp} / \mathrm{mm})$ and Schick 33 by $(4.238 \mathrm{lp} / \mathrm{mm})$ improved the resolution when highly sharpened. The SNR ratio was affected by sharpening. Some general trends emerged indicating the SNR ratio was decreasing when sharpening was used. A lower SNR is equivalent to an increase in noise. However atypical patterns were also observed. For example, the SNR for XDR and Schick 33 increased for the baseline images as the exposure time increased. In addition, the SNR for PSP fluctuated and did not demonstrate an overall trend for baseline images. Sharpening increased noise at all levels apart from highly sharpened for XDR where it returned to baseline levels. However, visibly all modalities demonstrated an appearance of increased noise after sharpening. This topic will be discussed further in chapter four.

The percent of overshoot was calculated using the percent change in gray value at the margins when compared to the baseline image. With moderate sharpening we saw a $1 \%$ change with XDR, 3\% for PSP and $12 \%$ for Schick 33 . When highly sharpened the change in gray value was 7\% for PSP, $9 \%$ for XDR and 31\% for Schick 33. (Table 2.) Conventional film was omitted from the study due to the very poor quality of the baseline images once digitized. 
Table 2. Average Percent Overshoot

\section{Average \% of change in gray value: Overshoot, "halo' artifact}

\section{Moderate High Sharpening Sharpening}

$X D R$

$1 \%$

$9 \%$

Schick 33

$12 \%$

$31 \%$

PSP

$3 \%$

$7 \%$ 


\section{CHAPTER FOUR}

\section{DISCUSSION}

Digital radiographic imaging is an important tool for dental diagnosis. The technology continues to improve as it is expanding from 2 dimensional into 4 dimensional modalities (i.e. 3-D time series data). Education becomes as important as the knowledge of the capabilities and limitations of these technologies in a clinical environment. Understanding the capabilities and limitations of sharpening and other image processing tools beyond the general term of "enhancements" is important for those using the technology for oral diagnosis. Each manufacturer has their own proprietary and unknown process for creating a digital radiograph. This begins with how the image is captured, digitized, stored and then displayed.

Conventional film was omitted from the study due to the very poor quality of the baseline images after digitization. Resolution of conventional film is excellent. However, during the digitization process this characteristic was lost. Even optimally exposed images consistently performed poorly using the criteria outlined for measuring resolution, negating the reason for its inclusion. This performance highlights the limitations when digitizing conventional film including but not limited to: operator error, hardware performance, and dissimilarity in technologies. Only the discernibility of the human eye of these various test gauges is the best tool for evaluating film images. Although improvement in resolution was observed, this was not uniform in all cases. This improvement carried with it an increase in noise and artifact. Increased overshoot was observed with increased sharpening. The Schick 33 sensor, which demonstrated the greatest improvement in resolution, also demonstrated the most overshoot. Increased 
artifact increases the possibility for misdiagnoses of oral conditions. (Brettle, 2011) (Schweitzer, 2010) (Liedke, 2015)

Although the SNR values are significant, both the XDR and Schick 33 sensors had atypical patterns with increased exposure. As mentioned, visibly all modalities demonstrated an appearance of increased noise after moderate and high sharpening which numerically was not always reflected. For the purpose of this study, the SNR was calculated without a raw image. In the true engineering sense, SNR should properly be calculated using the original raw data from the sensor but raw data is usually unavailable from the manufacturer. The calculation for SNR did not take into account two image manipulations that cannot be determined due to either patent protection or industry privacy; 1) the inversion of the gray scale that occurs before being displayed causing the SNR numbers to reverse and, 2) the functional transformation of the original sensor data due to vendors proprietary processing procedures. In order to correct this in future studies, a true confirmation should be completed on the raw data that would require further manufacture corroboration due to varying proprietary steps in processing.

Dynamic range was the least affected by sharpening followed by contrast resolution. In part this can be explained by the larger sampling area presented by both the step wedge and the wells.

The limitations for this study include the number of variables which were so large that it limited the number of samples that one could obtain and analyze for each element of the test matrix. The modified Mah protocol used for analysis is not ideal because it is partly subjective. Although strict guidelines were developed and adhered to, it relies occasionally on operator judgment and constantly on subjective visual perception. Two 
examples of this involved sampling and analysis. With sampling some of the image landmarks become more difficult to identify outside the ideal exposure range. While analyzing the plot profiles, the noise at times hindered identifying whether an object was truly there or an aberration.

More dental industry standards are needed to overcome the proprietary aspect of varying manufacture's image processing protocols. Image manipulation occurs at various levels across all radiographic imaging modalities. A varying degree of processing control is given to the clinician end user (dentists and allied clinical personnel), while some manufacturers set and lock these features omitting the clinician entirely.

\section{Conclusions}

Within the limitations of this study, the following conclusion can be made: Image sharpening to radiographic images creates artifacts that can be misinterpreted. Image sharpening of radiographic images significantly affects resolution, radiographic noise and overshoot. No significant effect was seen on dynamic range and contrast resolution. Objective ways to analyze contrast and spatial resolution are difficult to implement by an average dental professional. While sharpening can be a useful tool, accurate radiographic diagnosis is best achieved by looking at both the sharpened and unsharpened image then attempting to evaluate the degree of change in the anatomic outlines. This should be confirmed with clinical examination. In addition, phantoms provide a critical method to objectively evaluate the performance of sensors. 


\section{REFERENCES}

Affairs, A. D. (2006). The use of dental radiographs: update and recommendations. The Journal of the American Dental Association , 137 (9), 1304-1312.

Brettle, D. \&. (2011). The impact of digital image processing artefacts mimicking pathological features associated with restorations. British dental journal, 211 (4), 167-170.

Dexis. (2017, May 26). Digital X-ray \& Imaging For Dental Professionals. Retrieved May 26, 2017, from Dexis: http://www.dexis.com/sensor

Drost, W. T. (2008). Digital radiography artifacts. Veterinary radiology \& ultrasound, 49 (s1), S48-S56.

Herrmann, T. L. (2012). Best practices in digital radiography. Radiologic technology , 84 (1), 83-89.

Jacobsohn, P. H., \& Fedran, R. (1995). Making darkness visible: the discovery of X-ray and its introduction to dentistry. The Journal of the American Dental Association , $126(20), 1359-1366$.

Jiménez, D. A. (2009). Digital radiographic artifacts. Veterinary Clinics of North America: Small Animal Practice, 39 (4), 689-709.

Lança, L. \&. (2009). Digital radiography detectors-A technical overview: Part 2. Radiography, 15 (2), 134-138.

Liedke, G. S.-N. (2015). Diagnostic accuracy of conventional and digital radiography for detecting misfit between the tooth and restoration in metal-restored teeth. The Journal of prosthetic dentistry, 113 (1), 39-47.

Mah, P. M. (2011). Quality assurance phantom for digital dental imaging. Oral Surgery, Oral Medicine, Oral Pathology, Oral Radiology, and Endodontology. Oral Surgery, Oral Medicine, Oral Pathology, Oral Radiology, and Endodontology, $112(5), 632-639$.

Oestmann, J. W. (1991). Hardware and software artifacts in storage phosphor radiography. Radiographics , 11 (5), 795-805.

Raper, H. R. (1953). Notes on the early history of radiodontia: With special attention to its relation to the Indiana university school of dentistry. Oral Surgery, Oral Medicine, Oral Pathology , 6 (1), 70-81.

Reinhard, E. H. (2010). High dynamic range imaging: acquisition, display, and imagebased lighting. Morgan Kaufmann . 
Russ, J. C. (2016). The image processing handbook. Journal of Computer Assisted Tomography. CRC press .

Samei, E. (2003). Performance of digital radiographic detectors: quantification and assessment methods. Advances in digital radiography: RSNA , 37-47.

Sansare, K. K. (2014). Early victims of X-rays: a tribute and current perception. Dentomaxillofacial Radiology .

Schweitzer, D. M. (2010). A digital radiographic artifact: A clinical report. The Journal of prosthetic dentistry, 103 (6), 326-329.

Solomon, S. L. (1991). Artifacts in computed radiography. American journal of roentgenology, 157 (1), 181-185. .

Tan, T. H. (1997). Uberschwinger artefact in computed radiographs. The British journal of radiology , 70 (832), 431-431.

Udupa, H. M. (2013). Evaluation of image quality parameters of representative intraoral digital radiographic systems. Oral surgery, oral medicine, oral pathology and oral radiology, $116(6), 774-783$.

Versteeg, C. H. (1997). Efficacy of digital intra-oral radiography in clinical dentistry. Journal of dentistry , 25 (3), 215-224.

Walker, T. F. (2013). Digital intraoral radiographic quality assurance and control in private practice. General dentistry, 62 (5), 22-29.

Wenzel, A. (1998). Digital radiography and caries diagnosis. Dentomaxillofacial Radiology, 27 (1), 3-11.

Williams, M. B. (2007). Digital radiography image quality: image acquisition. Journal of the American College of Radiology , 4 (6), 371-388. 